\author{
M. S. Christiansen · E. Hommel · E. Magid • \\ B. Feldt-Rasmussen
}

\title{
Orosomucoid in urine is a powerful predictor of cardiovascular mortality in normoalbuminuric patients with type 2 diabetes at five years of follow-up
}

Received: 9 June 2004 / Accepted: 13 August 2004 / Published online: 11 January 2005

C) Springer-Verlag 2005

\begin{abstract}
Aims/hypothesis: To study whether urinary orosomucoid excretion rate (UOER) predicts mortality in normoalbuminuric patients with diabetes at 5 years of follow-up, and to investigate the relationship between orosomucoid in serum and urine. Methods: A cohort of 578 patients with diabetes (430 type 2, 148 type 1) was followed prospectively for an average of 5 years. UOER was measured in timed overnight urine samples. Results: Eightytwo patients with type 2 diabetes and 17 patients with type 1 diabetes died. Among patients with type 2 diabetes, 251 (58\%) had normoalbuminuria; increased UOER independently predicted cardiovascular mortality (OR 4.94, 95\% CI $1.60-15.22 ; p<0.006)$ in those with normoalbuminuria and in the entire cohort of patients with type 2 diabetes (odds ratio 3.63, 95\% CI $1.50-8.81 ; p<0.005)$. Patients with increased UOER had a higher all-cause mortality than those with normal UOER (log-rank test, $p<0.001$ for type 2 patients; $p<0.04$ for type 1 patients). In patients with type 1 diabetes, there were five cardiovascular deaths and no significant predictive value of UOER. Patients with increased UOER had a subclinical increase in serum orosomucoid. Conclusion/interpretation: Increased UOER was an independent, powerful predictor of cardiovascular mortality in normoalbuminuric patients with type 2 diabetes and in the entire cohort of patients with type 2 diabetes. There were indications of UOER as being a valuable marker in type 1
\end{abstract}

\footnotetext{
M. S. Christiansen $(\bowtie) \cdot$ E. Magid

Department of Clinical Biochemistry, Amager Hospital,

Italiensvej 1

2300 Copenhagen, Denmark

e-mail: skovdal@dadlnet.dk

Tel.: +45-3234-3710

Fax: +45-3234-3997

E. Hommel

Steno Diabetes Centre,

Gentofte, Denmark

B. Feldt-Rasmussen

Department of Nephrology and Endocrinology, Rigshospitalet,

Copenhagen, Denmark
}

diabetes that showed differences in survival between patients with normal versus increased UOER. Serum orosomucoid was associated with UOER; UOER may be a marker of lowgrade inflammation in patients with diabetes.

Keywords All-cause mortality · Cardiovascular mortality · Inflammation - Microalbuminuria - Type 2 diabetes · Type 1 diabetes $\cdot$ Urinary orosomucoid excretion

Abbreviations IQR: Inter-quartile range - UAER: Urinary albumin excretion rate · UOER: Urinary orosomucoid excretion rate

\section{Introduction}

Patients with type 1 and type 2 diabetes have a high mortality compared with the background population. This is primarily caused by a high cardiovascular morbidity and mortality [1-4].

Urinary orosomucoid excretion rate (UOER) was demonstrated to predict all-cause and cardiovascular mortality independently of classic risk factors in a 2.4-year followup study of patients with type 2 diabetes including patients with normo-, micro- and macroalbuminuria [5]. The study was not powered to show predictive value of UOER in the subgroup of patients with normal urinary albumin excretion rate (UAER); but still increased UOER was strongly associated with cardiovascular mortality in the normoalbuminuric subgroup. Another limitation of the study was the lack of access to serum values of orosomucoid [5].

Orosomucoid ( $\alpha_{1}$-acid glycoprotein) is a single-chain polypeptide [6] mainly produced in the liver cells and the plasma concentration increases in response to inflammation and other stressful stimuli [7]. In vitro and in vivo studies have shown that orosomucoid has immunomodulatory and anti-inflammatory effects, thereby protecting against tissue damage from excessive inflammation $[7,8]$.

Clinical and experimental studies have suggested that an inflammatory process is involved in the development of 
atherosclerosis [9] and type 2 diabetes [10]. Subclinical elevations of orosomucoid in serum have been found in patients with type 2 diabetes [11, 12]; this may be a sign of a chronic low-grade inflammation [13]. We hypothesize that there is a link between a systemic inflammatory response and increased UOER.

The aims of our study were to investigate if increased UOER predicts mortality in the subgroup of patients with normoalbuminuria as well as in the entire cohort of patients with type 2 and type 1 diabetes at 5 years of follow-up. We also investigated the relation between serum orosomucoid and UOER to evaluate the level of systemic inflammatory response in patients with increased UOER.

\section{Subjects and methods}

Participants A cohort study was performed from September 1995 to December 1998 consecutively including all patients who attended the outpatient clinic at Amager Hospital, Copenhagen with type 2 and type 1 diabetes and dipstick negative overnight urine samples. Four hundred and thirty patients with type 2 diabetes and 148 patients with type 1 diabetes were followed until death, emigration or 31 December 2002. The diagnosis of type 2 diabetes was based on the World Health Organization criteria (1985) (fasting blood glucose $\geq 6.7 \mathrm{mmol} / \mathrm{l}$ or 2 -h OGTT values of capillary blood glucose $\geq 11.1 \mathrm{mmol} / 1$ and not being prone to keto-acidosis). The diagnosis of type 1 diabetes was based on the same criteria, but patients were prone to keto-acidosis, and fasting serum C-peptide values (if available) were less than $200 \mathrm{pmol} / \mathrm{l}$. Duration of diabetes was defined as duration from the date of diagnosis. Medical history was obtained from medical records. The registration of clinical parameters is described in detail elsewhere [5]. We registered the presence or absence of cardiovascular diseases (hypertension, coronary heart disease, previous myocardial infarction, stroke and congestive heart failure), diabetic retinopathy, smoking habits and medication (use of insulin and ACE inhibitors).

Information on causes of death was gathered through the central Danish Registry of Causes of Death and through death certificates from journal files and registered according to the International Classification of Diseases (ICD 10).

Blood and urine analyses Analyses of blood and urine are described in detail elsewhere [5]. Data on $\mathrm{HbA}_{1} \mathrm{c}$ and plasma creatinine were collected from routine blood analyses performed on fresh samples within a period of maximum 6 months before or after the corresponding urine sample. In a subgroup of patients we determined orosomucoid in serum and urine collected within $24 \mathrm{~h}$. Orosomucoid in serum was analysed on a Cobas Integra 700 (Roche, Basel, Switzerland) by immunoturbidimetry. The reference interval for orosomucoid in serum was $0.45-1.17 \mathrm{~g} / \mathrm{l}$.

Urinary orosomucoid and albumin Urine samples were collected as timed overnight samples, stored at $2-8^{\circ} \mathrm{C}$ without any additives and analysed within a week of col- lection. Urinary orosomucoid and albumin were analysed using an in-house method by immunoturbidimetry (Cobas Mira, Roche, Basel, Switzerland). Detection limits and intraseries imprecision $(\mathrm{CV} \%)$ were: urinary orosomucoid 1.2 $\mathrm{mg} / \mathrm{l}(5.6 \%)$ and urinary albumin $3.3 \mathrm{mg} / \mathrm{l}(3.0 \%)$. Cut-off values were: UOER $>0.88 \mu \mathrm{g} / \mathrm{min}$ [14] and UAER $>20 \mu \mathrm{g} /$ min [15]. Microalbuminuria was defined as UAER of $20-200 \mu \mathrm{g} / \mathrm{min}$ in two of three urine samples. Macroalbuminuria was defined as UAER $>200 \mu \mathrm{g} / \mathrm{min}$ [15].

Ethics The local ethics committee in Copenhagen approved the study protocols (KF 01- 403/97 and KF 01-404/97).

Statistics Statistical analyses were performed using Statistica for Windows version 6 (StatSoft Inc., Tulsa, OK, USA). Normal distribution of continuous variables was tested by the Kolmogorov-Smirnov test. Two sample $t$-test was used for the comparison of normal distributed continuous variables and Mann-Whitney $U$-test was used for non-normal distributed continuous variables. Chi-square test with Yates' correction or Pearson chi-square test was used for dichotomous variables. Nonparametric correlations were performed with Spearman rank order correlation. Many patients had urinary orosomucoid concentrations below the detection limit of the assay. Therefore, we made this parameter a dichotomous variable in multivariate analysis. The median values and correlations of urinary orosomucoid were calculated for patients with increased values of UOER. Survival analyses were done using the method of Kaplan-Meier and survival curves were compared by log-rank test. Cox proportional hazards regression analysis with backward stepwise regression was used for multivariate analysis. Unless otherwise stated the multivariate analysis included all baseline variables except retinopathy and smoking due to many missing values. Furthermore, patients with missing values were excluded from the multivariate analysis. Logarithm transformed values of UAER ( $\ln$ UAER) were used in the multivariate analysis because of a positive skewness of distribution.

Values below the detection limit of urinary orosomucoid were fixed at $1.19 \mathrm{mg} / \mathrm{l}$ for the calculation of clearance of orosomucoid. Clearance values of orosomucoid were calculated as: [urine orosomucoid concentration $(\mathrm{mg} / \mathrm{l})$ times urine volume $(\mathrm{ml})] /[$ serum orosomucoid concentration $(\mathrm{g} / \mathrm{l})$ times urine collection time $(\mathrm{min})]$.

A $p$ value of less than 0.05 was considered significant.

\section{Results}

A cohort of 430 patients with type 2 diabetes and one of 148 patients with type 1 diabetes were followed for a mean $\pm \mathrm{SD}$ (range) period of $4.9 \pm 1.6(0.01-7.2)$ and $5.3 \pm 1.3$ (0.09-7.2) years, respectively. One patient with type 2 diabetes and five patients with type 1 diabetes emigrated. No patients were lost to follow-up.

Patients with type 2 diabetes Patients with type 2 diabetes (273 men/157 women) had a mean \pm SD age of $59 \pm 11$ 
years, $\mathrm{HbA}_{1} \mathrm{c}$ of $8.4 \pm 1.9 \%$, and median (range) duration of diabetes of $3.0(0.02-37)$ years. The patients were subclassified in relation to normal or increased UOER; anthropometrical and clinical data of these subgroups are shown in Table 1 . At baseline, patients with type 2 diabetes and increased UOER were older; were more often men; had a longer duration of diabetes; a higher level of $\mathrm{HbA}_{1} \mathrm{c}$, plasma creatinine and UAER; were heavier; had a higher prevalence of hypertension, heart failure and retinopathy; and were more frequently treated with ACE inhibitors than patients with normal UOER.

Median (IQR) value of UOER was $10.7(5.4-22.0) \mu \mathrm{g} /$ min for patients with increased UOER $(n=242)$. The median increase in UOER compared with the cut-off value was 12-fold for patients with increased UOER. The prevalence of microalbuminuria was $130(30 \%)$ and that of macroalbuminuria was 49 (11\%).

A total of $82(19 \%)$ patients with type 2 diabetes died in the follow-up period. The causes of death are listed in Table 2 .

In the subgroup of patients with normoalbuminuria $(n=251), 21$ patients died of cardiovascular diseases. In this subgroup, seven $(5 \%)$ patients with normal UOER $(n=152)$ and $14(14 \%)$ patients with increased UOER $(n=99)$ died of cardiovascular diseases in the follow-up period. A signif-
Table 2 Causes of death

\begin{tabular}{lcl}
\hline & Type 2 patients & Type 1 patients \\
\hline Cardiovascular diseases, $n$ & 45 & 5 \\
Unknown, $n$ & 13 & 6 \\
Cancer, $n$ & 9 & 2 \\
Infections, $n$ & 6 & 2 \\
Other causes, $n$ & $9^{\mathrm{a}}$ & $2^{\mathrm{b}}$ \\
\hline
\end{tabular}

${ }^{\text {a } U r a e m i a ~}(n=3)$, type 2 diabetes $(n=2)$, multiorgan failure $(n=2)$, hepatic coma $(n=1)$, suicide $(n=1)$

${ }^{\mathrm{b}}$ Hepatic coma $(n=1)$; accident $(n=1)$

icant difference in survival was seen between the subgroups (log-rank test: $p<0.007$ ) as shown in Fig. 1. In multivariate analysis, UOER independently predicted cardiovascular mortality with an odds ratio of 4.94 (95\% CI: 1.60-15.22; $p<0.006)$ in the subgroup of patients with normoalbuminuria as seen in Table 3.

In the entire cohort of patients with type 2 diabetes, nine (5\%) patients with normal UOER and $36(15 \%)$ patients with increased UOER died of cardiovascular diseases in the follow-up period. A significant difference in survival in relation to cardiovascular mortality was seen between the

Table 1 Anthropometrical and clinical data at baseline for patients with type 2 and type 1 diabetes subgrouped by normal versus increased urinary orosomucoid excretion rates (UOER)

\begin{tabular}{|c|c|c|c|c|c|c|}
\hline & \multicolumn{3}{|l|}{ Type 2} & \multicolumn{3}{|l|}{ Type 1} \\
\hline & Normal UOER & Increased UOER & $p$-Value & Normal UOER & Increased UOER & $p$-Value \\
\hline Number (men/women) & 97/91 & $176 / 66$ & $<0.001^{\mathrm{a}}$ & $56 / 31$ & $35 / 26$ & $0.49^{\mathrm{a}}$ \\
\hline Age $(\text { years })^{b}$ & $57 \pm 11$ & $60 \pm 11$ & $<0.003^{\mathrm{c}}$ & $38 \pm 13$ & $44 \pm 19$ & $0.11^{\mathrm{c}}$ \\
\hline Duration of diabetes (years) ${ }^{\mathrm{d}}$ & $3(0.02-30)$ & $4(0.02-37)$ & $<0.04^{\mathrm{c}}$ & $7(0.02-32)$ & $7(0.02-66)$ & $0.12^{\mathrm{c}}$ \\
\hline Blood $\mathrm{HbA}_{1} \mathrm{c}(\%)^{\mathrm{b}}$ & $8.1 \pm 1.9$ & $8.6 \pm 1.8$ & $<0.002^{\mathrm{c}}$ & $8.4 \pm 1.8$ & $8.5 \pm 1.6$ & $0.52^{\mathrm{c}}$ \\
\hline Plasma creatinine $(\mu \mathrm{mol} / \mathrm{l})^{\mathrm{b}}$ & $71 \pm 20^{\mathrm{f}}$ & $85 \pm 32^{\mathrm{g}}$ & $<0.001^{\mathrm{c}}$ & $70 \pm 20^{\mathrm{h}}$ & $78 \pm 26^{\mathrm{i}}$ & $<0.02^{\mathrm{c}}$ \\
\hline $\operatorname{UAER}(\mu \mathrm{g} / \mathrm{min})^{\mathrm{d}}$ & $11(3-421)$ & $29(3-3,436)$ & $<0.001^{\mathrm{c}}$ & $13(2-81)$ & $194(2-2,461)$ & $<0.001^{\mathrm{c}}$ \\
\hline Systolic BP (mmHg) ${ }^{\mathrm{b}}$ & $144 \pm 23^{j}$ & $148 \pm 23^{\mathrm{i}}$ & $0.06^{\mathrm{c}}$ & $131 \pm 20$ & $134 \pm 20$ & $0.28^{\mathrm{c}}$ \\
\hline Diastolic BP $(\mathrm{mmHg})^{\mathrm{b}}$ & $84 \pm 12^{\mathrm{j}}$ & $85 \pm 13^{\mathrm{i}}$ & $0.28^{\mathrm{c}}$ & $78 \pm 11$ & $78 \pm 11$ & $0.59^{\mathrm{c}}$ \\
\hline Weight $(\mathrm{kg})^{\mathrm{b}}$ & $84 \pm 17^{\mathrm{k}}$ & $88 \pm 17^{1}$ & $<0.01^{\mathrm{c}}$ & $74 \pm 15^{\mathrm{m}}$ & $74 \pm 14^{\mathrm{n}}$ & $0.85^{\mathrm{c}}$ \\
\hline Hypertension (\%) & 37 & 54 & $<0.001^{\mathrm{a}}$ & 13 & 23 & $0.15^{\mathrm{a}}$ \\
\hline Heart failure $(\%)$ & 5 & 16 & $<0.001^{\mathrm{a}}$ & $1^{\mathrm{o}}$ & 5 & $0.39^{\mathrm{a}}$ \\
\hline Coronary heart disease (\%) & 19 & 21 & $0.63^{\mathrm{a}}$ & 3 & 5 & $0.98^{\mathrm{a}}$ \\
\hline Previous MI $(\%)$ & 12 & 14 & $0.68^{\mathrm{a}}$ & 1 & 0 & $0.86^{\mathrm{a}}$ \\
\hline Stroke $(\%)$ & 5 & 8 & $0.22^{\mathrm{a}}$ & 1 & 5 & $0.38^{\mathrm{a}}$ \\
\hline Retinopathy, simplex/proliferative (\%) & $14 / 1^{\mathrm{p}}$ & $19 / 4^{\mathrm{q}}$ & $<0.05^{\mathrm{e}}$ & $19 / 4^{\mathrm{r}}$ & $29 / 3^{1}$ & $0.38^{\mathrm{e}}$ \\
\hline Current smoking $(\%)$ & $43^{\mathrm{s}}$ & $45^{\mathrm{t}}$ & $0.75^{\mathrm{a}}$ & $63^{\mathrm{u}}$ & $63^{v}$ & $0.89^{\mathrm{a}}$ \\
\hline Insulin treatment $(\%)$ & 32 & 34 & $0.83^{\mathrm{a}}$ & 100 & 100 & - \\
\hline ACE inhibitor treatment (\%) & 34 & 50 & $<0.001^{\mathrm{a}}$ & 13 & 36 & $<0.002^{\mathrm{a}}$ \\
\hline Follow-up time (years) ${ }^{\mathrm{b}}$ & $5.2 \pm 1.3$ & $4.7 \pm 1.7$ & $<0.04^{\mathrm{c}}$ & $5.4 \pm 1.2$ & $5.2 \pm 1.4$ & $0.36^{\mathrm{c}}$ \\
\hline
\end{tabular}

UAER urinary albumin excretion rate, $M I$ myocardial infarction

${ }^{\mathrm{a}}$ Chi-square test, Yates' correction

${ }^{\mathrm{b}}$ Mean (SD)

${ }^{c}$ Mann-Whitney $U$-test

${ }^{\mathrm{d}}$ Median (range)

${ }^{\mathrm{e}}$ Pearson chi-square

$n$ corrected for missing values: ${ }^{\mathrm{f}} 183,{ }^{\mathrm{g}} 238,{ }^{\mathrm{h}} 73,{ }^{\mathrm{i}} 53,{ }^{\mathrm{j}} 186,{ }^{\mathrm{k}} 182,{ }_{2}{ }_{232},{ }^{\mathrm{m}} 83,{ }^{\mathrm{n}} 58,{ }^{\mathrm{o}} 86,{ }^{\mathrm{p}} 207,{ }^{\mathrm{q}} 81,{ }^{\mathrm{r}} 161,{ }^{\mathrm{s}} 166,{ }^{\mathrm{t}} 222,{ }^{\mathrm{u}} 71,{ }^{\mathrm{v}} 54$ 
Fig. 1 Kaplan-Meier survival curves in relation to cardiovascular mortality in normoalbuminuric patients with type 2 diabetes and normal (continuous straight line, $n=152)$ versus increased (broken line, $n=99$ ) urinary orosomucoid excretion rates at baseline; $p<0.007$ (logrank test)

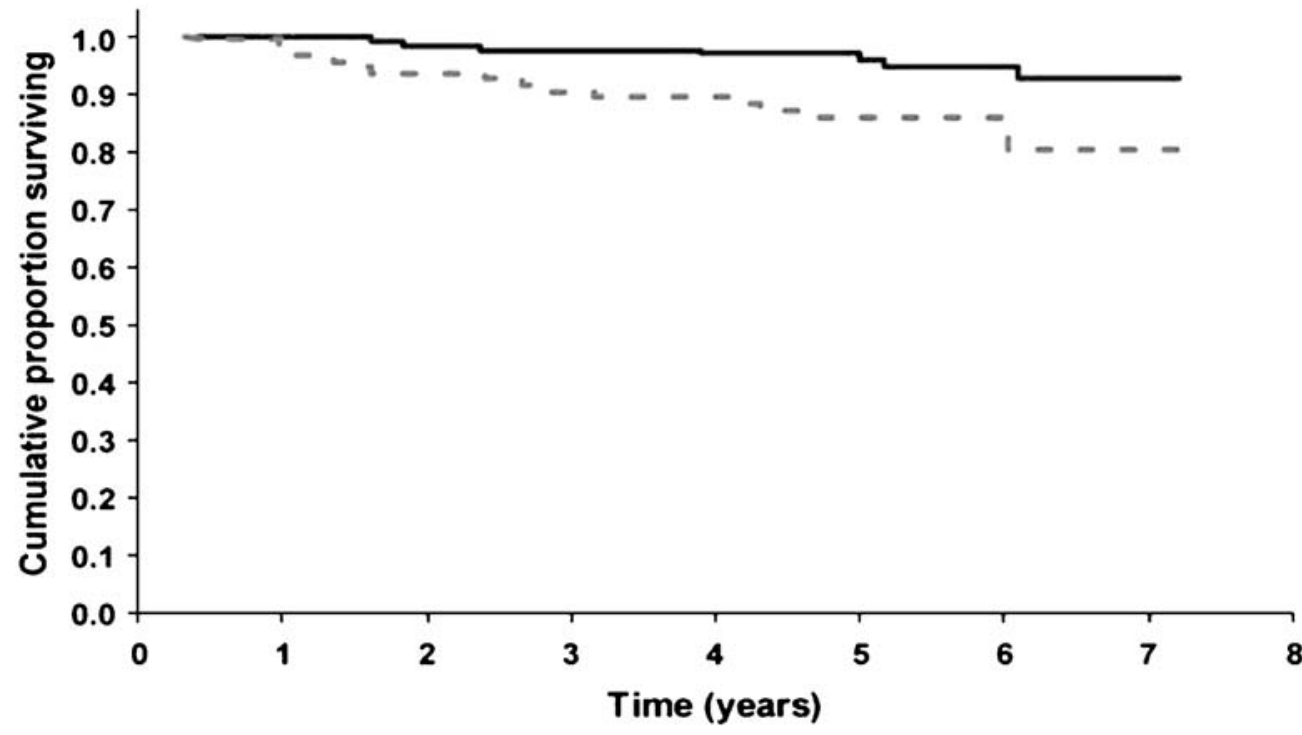

subgroups (log-rank test: $p<0.001)$. In multivariate analysis UOER independently predicted cardiovascular mortality with an odds ratio of $3.63(95 \%$ CI: $1.50-8.81 ; p<0.005)$ as seen in Table 4 . In the primary multivariate analysis, UAER was entered as a continuous variable. To evaluate if lowering of the cut-off value for UAER would change these results, we thereafter used UAER as a dichotomous variable with different cut-off values $(7,10,15$ or $20 \mu \mathrm{g} / \mathrm{min})$. Using different cut-off values for UAER did not change the results of the multivariate analysis of cardiovascular mortality.

A significant difference in survival in relation to all-cause mortality was seen between patients with normal versus increased UOER (log-rank test: $p<0.001)$. In multivariate analysis of all-cause mortality age (OR 1.06, 95\% CI 1.04 $-1.09 ; p<0.001$ ), ln UAER (OR 1.21, 95\% CI 1.05-1.40; $p<0.008$ ), plasma creatinine (OR 1.02, 95\% CI 1.01-1.02; $p<0.001$ ), history of coronary heart disease (OR 2.03, 95\% CI $1.26-3.27 ; p<0.004)$ and stroke (OR 2.82, 95\% CI 1.46 $-5.46 ; p<0.003)$ were independent predictors. When UAER was not included in the multivariate analysis, increased UOER independently predicted all-cause mortality with an OR of 1.80 (95\% CI 1.06-3.08; $p<0.04)$.

Table 3 Multiple regression analysis of cardiovascular mortality, patients with type 2 diabetes and normoalbuminuria $(n=251)$

\begin{tabular}{llc}
\hline Variable & $\begin{array}{l}\text { Hazard ratio } \\
(95 \% \mathrm{CI})\end{array}$ & $p$-Value \\
\hline Age (years) & $1.07(1.01-1.13)$ & $<0.03$ \\
$\begin{array}{l}\text { Coronary heart } \\
\text { disease (no/yes) }\end{array}$ & $3.39(1.28-8.99)$ & $<0.02$ \\
$\begin{array}{l}\text { Treatment with ACE } \\
\text { inhibitor (no/yes) }\end{array}$ & $3.36(1.26-8.98)$ & $<0.02$ \\
UOER (normal/increased) & $4.94(1.60-15.22)$ & $<0.006$ \\
\hline
\end{tabular}

Data are for selected patients $(n=235$, patients with missing values are excluded)
Orosomucoid in serum was not measured routinely in all patients. To explore whether a relationship exists between the concentration of orosomucoid in serum and UOER, we identified a subgroup of patients in whom orosomucoid had been determined in samples of blood and urine obtained within $24 \mathrm{~h}$. This subgroup was similar to the total group regarding all clinical variables recorded, with the exception of a lower plasma creatinine $(p<0.02)$ in patients with type 1 diabetes. Patients were reclassified in accordance with the new calculation of UOER for comparison with serum orosomucoid values. In the representative subgroup of patients with type 2 diabetes $(n=56)$ there were significantly higher serum orosomucoid concentrations in patients with increased UOER $(n=34)$ compared with patients with normal UOER $(n=22)$. The mean \pm SD (range) serum orosomucoid was $0.94 \pm 0.20(0.57-1.35) \mathrm{g} / 1$ versus $0.78 \pm 0.22$ $(0.46-1.42) \mathrm{g} / \mathrm{l}, p<0.005$. One patient with normal UOER and four patients with increased UOER had a slightly elevated serum orosomucoid value. All remaining values were within the reference range. Clearance of orosomucoid was higher in patients with increased UOER $(n=34)$ compared with patients with normal UOER $(n=22)$ (median (range) orosomucoid clearance: $8.3(2.0-177.6) \mu \mathrm{l} / \mathrm{min}$ versus 2.0 $(0.4-4.5) \mu \mathrm{l} / \mathrm{min}, p<0.001)$.

Table 4 Multiple regression analysis of cardiovascular mortality, patients with type 2 diabetes

\begin{tabular}{lll}
\hline Variable & Hazard ratio $(95 \% \mathrm{CI})$ & $p$-Value \\
\hline Age (years) & $1.06(1.02-1.10)$ & $<0.002$ \\
Plasma creatinine $(\mu \mathrm{mol} / \mathrm{l})$ & $1.02(1.01-1.03)$ & $<0.003$ \\
Coronary heart disease (no/yes) & $5.85(2.93-11.69)$ & $<0.001$ \\
Stroke (no/yes) & $2.36(1.02-5.44)$ & $<0.05$ \\
UOER (normal/increased) & $3.63(1.50-8.81)$ & $<0.005$ \\
\hline
\end{tabular}

Data are for selected patients $(n=402$, patients with missing values are excluded) 
Table 5 Multiple regression analysis of all-cause mortality, patients with type 1 diabetes

\begin{tabular}{llc}
\hline Variable & Hazard ratio $(95 \% \mathrm{CI})$ & $p$-Value \\
\hline Age (years) & $1.07(1.04-1.10)$ & $<0.001$ \\
$\ln$ UAER $(\mu \mathrm{g} / \mathrm{min})$ & $1.49(1.13-1.96)$ & $<0.005$ \\
\hline
\end{tabular}

Data are for selected patients $(n=126$, patients with missing values are excluded)

Patients with type 1 diabetes Patients with type 1 diabetes (91 men $/ 57$ women) had a mean \pm SD age of $40 \pm 16$ years, $\mathrm{HbA}_{1} \mathrm{c}$ of $8.4 \pm 1.7 \%$, and median (range) duration of diabetes of $7.0(0.02-66)$ years. The differences between the two subgroups with normal versus increased UOER were plasma creatinine, UAER and the prevalence of treatment with ACE inhibitors, as seen in Table 1.

Median (IQR) values of UOER were $7.8(3.8-19.4) \mu \mathrm{g} /$ min for patients with increased UOER $(n=61)$. The median increase in UOER compared with the cut-off value was 9fold for patients with increased UOER.

Increased UOER was correlated to UAER $(r=0.50, p<0.01$; $n=61)$ and plasma creatinine $(r=0.28, p<0.05 ; n=61)$. Age and $\mathrm{HbA}_{1} \mathrm{c}$ were not correlated to increased UOER. The prevalence of microalbuminuria was $39(26 \%)$ and that of macroalbuminuria was nine $(6 \%)$.

A total of $17(11 \%)$ patients with type 1 diabetes died in the follow-up period. The causes of death are listed in Table 2. Six (7\%) patients with normal UOER and 11 (18\%) patients with increased UOER died in the follow-up period. A significant difference in survival was seen between the subgroups (log-rank test, $p<0.04)$ in relation to all-cause mortality. As seen in Table 5, ln UAER and age independently predicted all-cause mortality. The multivariate analysis of all-cause mortality included sex, age, duration of diabetes, history of hypertension, coronary heart disease, stroke, treatment with $\mathrm{ACE}$ inhibitors, $\mathrm{HbA}_{1} \mathrm{c}$, plasma creatinine, In UAER, classification of normal or increased UOER, and systolic and diastolic blood pressure.

Only five patients with type 1 diabetes died of cardiovascular diseases. In multivariate analysis of cardiovascular mortality, age [OR 1.11 (95\% CI 1.03-1.20; $p<0.008)$ ] and coronary heart disease [OR $13.64(95 \%$ CI 1.71 $-108.96 ; p<0.02)]$ were independent predictors in a model including sex, age, coronary heart disease, ln UAER and classification of normal or increased UOER.

In the subgroup of patients with type 1 diabetes who had corresponding serum and urine orosomucoid analysis $(n=17)$ there were significantly higher values of serum orosomucoid in those with increased UOER $(n=5)$ compared with those with normal UOER $(n=12)$. The mean \pm SD (range) serum orosomucoid was $0.90 \pm 0.08(0.78-1.01)$ versus 0.71 \pm 0.17 (0.47-1.03) g/l, $p<0.04$. No patients had serum orosomucoid values above the upper reference value. Clearance of orosomucoid was higher in patients with increased UOER compared with those with normal UOER (median (range) orosomucoid clearance: 7.4 (4.1-189.6) versus 1.8 (1.0-6.6) $\mu \mathrm{l} / \mathrm{min}, p<0.003)$.

\section{Discussion}

In the present study, we have shown for the first time that increased UOER is an independent, powerful predictor of cardiovascular mortality in normoalbuminuric patients with type 2 diabetes at 5 years of follow-up. We also confirmed our previous observation that increased UOER is an independent predictor of cardiovascular mortality in patients with type 2 diabetes and micro- and macroalbuminuria at 2.4 years of follow-up [5]. Increased UOER, therefore, seems a superior marker for cardiovascular mortality compared with microalbuminuria in patients with type 2 diabetes.

In patients with type 1 diabetes there was a significant difference in survival between patients with increased UOER compared with those with normal UOER. However, UOER was not an independent marker for all-cause or cardiovascular mortality. This might be explained by the low number $(n=5)$ of cardiovascular deaths in patients with type 1 diabetes in the follow-up period. Additionally, the cohort of patients with type 1 diabetes was younger and had a less adverse cardiovascular profile compared with the cohort with type 2 diabetes. We found no significant predictive value of UOER, presumably due to the low power of the smaller cohort of patients with type 1 diabetes; however, the results indicate that a longer follow-up time may yield significant results.

In the present study, we had access to serum values of orosomucoid in a representative subgroup of patients. Serum values of orosomucoid were slightly higher in patients with increased UOER compared with patients with normal UOER in both type 1 and type 2 diabetic patients. The majority of the serum orosomucoid values were within the normal range. This subclinical increase in serum orosomucoid in patients with increased UOER is indicative of a chronic inflammatory condition and not an acute phase response, which is usually characterised by a several-fold elevation of the initial level [7]. The association of elevated values of serum orosomucoid and UOER in subgroups of both type 2 and type 1 diabetic patients may reflect chronic low-grade inflammation as a common pathophysiological element of cardiovascular morbidity. A low-grade systemic inflammatory condition is hypothesized to precede the development of type 2 diabetes and atherosclerosis. In a population-based cohort study, subclinical elevations of serum orosomucoid and other inflammatory markers were predictive of the development of type 2 diabetes [13]. Subclinical elevations of serum orosomucoid have also been found in patients with type 2 diabetes [12] and were associated with development of cardiovascular disease and cardiovascular mortality in patients with type 2 diabetes in a combined inflammatory score model [16]. In a study of 40 patients with type 1 diabetes the plasma concentrations of orosomucoid were normal and comparable to healthy control persons. However, signs of chronic inflammation were found in diabetic patients where an increased amount of fucosylated orosomucoid was found by crossed immunoelectrophoresis [17]. The differences observed for orosomucoid in serum in our study occur within the reference interval. Likewise, the 
subclinical increase of inflammatory markers such as Creactive protein, which has been found to be an independent risk marker of mortality in epidemiological studies [18, 19], does not allow individual classification based on normal or elevated values. In contrast, the mean occurrence of UOER values 9-12 times higher than the upper reference value readily allows classification of individuals as either normal or abnormal regarding UOER. The pathophysiological mechanisms causing this dramatic amplification of a putative sign of low-grade inflammation are unknown. However, several possible explanations of the increased UOER can be listed. Overflow proteinuria could cause an increase in UOER; however, this phenomenon is unlikely to explain all of the variation in UOER as discussed below. There might be a selective increase in the glomerular permeability in patients with increased UOER accompanied by increased UAER, or there might be a renal secretion of orosomucoid causing the increased UOER. There might even be different mechanisms of UOER increase in normoalbuminuric and microalbuminuric patients.

In agreement with our results, a Japanese study found that UOER increased in parallel with UAER in patients with type 2 diabetes and that clearance of orosomucoid was highly correlated to UAER. They also found that a large subgroup of patients with normoalbuminuria had increased UOER [14]. We have confirmed these results. These data support a theory of increased glomerular permeability causing the increased UOER in patients with increased UAER. We believe that the increased UOER in the normoalbuminuric subgroup requires an alternative explanation such as renal secretion of orosomucoid.

From searching the PubMed database, we found no published data on urinary orosomucoid in patients with type 1 diabetes.

Clearance values of orosomucoid in the subgroup analyses were much higher in patients with increased UOER compared with those with normal UOER in both type 2 and type 1 diabetic patients. If increased serum values of orosomucoid had had a high impact on clearance values, the final result would have been a lowering of the clearance of orosomucoid. Thus the increased clearance values of orosomucoid were primarily caused by a high urinary excretion and probably unaffected by the serum concentrations of orosomucoid. In a study of patients with chronic renal failure, the clearance of orosomucoid was highly increased despite impaired renal function [20]. The patients with renal failure had increased serum concentrations of orosomucoid probably as a sign of chronic inflammation, but the UOER was not correlated to the serum concentration of orosomucoid. These findings are in accordance with the hypothesis of increased glomerular permeability in patients with increased UOER accompanied by micro- or macroalbuminuria. Furthermore, the lack of correlation between UOER and serum orosomucoid in patients with renal failure supports our considerations of increased UOER not simply being a result of overflow proteinuria.

The presence of micro- and macroalbuminuria predict all-cause mortality [19, 21-24], and microalbuminuria is an independent predictor of cardiovascular mortality in patients with diabetes $[22,23,25]$. At a lower cut-off value, urinary albumin was predictive of the development of ischemic heart disease [26] as well as cardiovascular mortality in the general population [27, 28]. This suggests that the predictive value of UAER is a continuum starting well below the recommended cut-off value for microalbuminuria. The effect of albuminuria on cardiovascular mortality in our cohort of patients with type 2 diabetes might be disclosed by lowering the cut-off value for UAER. However, we have shown that the predictive value of UOER on cardiovascular mortality in our study was independent of the cut-off value for UAER. We conclude that our data indicate a high predictive value of UOER for cardiovascular mortality independently of classic cardiovascular risk factors and urinary albumin level in patients with type 2 diabetes. As a consequence, we believe that patients with type 2 diabetes and increased UOER may be appropriate targets for intervention, but clinical intervention studies are required to clarify this issue.

The prevalence of microalbuminuria (26 and 30\%) and macroalbuminuria (6 and $11 \%$ ) in our study is comparable to other studies $[19,29,30]$. In a 5-year follow-up study of 328 patients with type 2 diabetes all-cause mortality was $16 \% ; 57 \%$ of these deaths were caused by cardiovascular diseases [31]. The patients in the referred study were of comparable age as our cohort of patients with type 2 diabetes. In our study, $19 \%$ of the patients with type 2 diabetes died in 5 years of follow-up and $55 \%$ of the deaths were caused by cardiovascular diseases. In addition, increased UOER was associated with increased prevalence of hypertension and heart failure in patients with type 2 diabetes in our study. Hypertension and heart failure are associated with development of microalbuminuria in patients with diabetes as well as in non-diabetic persons [32].

In this study we used fresh samples of urine for the analyses of proteins and we thereby avoided the well known problems of loss of proteins during storage at $-20^{\circ} \mathrm{C}$ $[33,34]$.

A limitation of this study is the access to cardiovascular history through journal files, rather than by classification of cardiovascular diseases by standardised investigations of each patient. Since the prevalence of cardiovascular diseases is high in patients with diabetes, silent myocardial ischaemia may have been overlooked and the number of diagnosed patients with cardiovascular diseases may have been underestimated.

Another limitation of the study is the use of UOER as a dichotomous rather than a continuous variable. However, in many epidemiological studies of microalbuminuria this variable is classified as a categorical variable $[21,22,35,36]$.

In conclusion, at 5 years of follow-up increased UOER was an independent, powerful predictor of cardiovascular mortality in normoalbuminuric patients with type 2 diabetes as well as in the entire cohort of patients with type 2 diabetes. The predictive value of UOER was independent of classic cardiovascular risk factors as well as urinary 
albumin level. The study was underpowered to show predictive value of UOER on mortality in patients with type 1 diabetes. However, UOER may be a valuable marker in type 1 diabetes as was also indicated by the differences in survival between patients with normal versus increased UOER.

We hypothesize that an increase in UOER in both type 1 and type 2 diabetes is caused by chronic low-grade inflammation. The possible therapeutic effect on cardiovascular morbidity and mortality of interventions targeted towards patients with increased UOER remains to be investigated.

Acknowledgements We are grateful to senior technologist Lise Foged, Amager Hospital, for technical assistance. The study was supported by Ernst and Vibeke Husman's Foundation.

\section{References}

1. Gu K, Cowie CC, Harris MI (1998) Mortality in adults with and without diabetes in a national cohort of the U.S. population, 1971-1993. Diabetes Care 21:1138-1145

2. Laing SP, Swerdlow AJ, Slater SD et al (2003) Mortality from heart disease in a cohort of 23,000 patients with insulin-treated diabetes. Diabetologia 46:760-765

3. Morrish NJ, Wang SL, Stevens LK, Fuller JH, Keen H (2001) Mortality and causes of death in the WHO multinational study of vascular disease in diabetes. Diabetologia 44:S14-S21

4. Roper NA, Bilous RW, Kelly WF, Unwin NC, Connolly VM (2002) Cause-specific mortality in a population with diabetes: South Tees Diabetes Mortality Study. Diabetes Care 25:43-48

5. Christiansen MS, Hommel E, Magid E, Feldt-Rasmussen B (2002) Orosomucoid in urine predicts cardiovascular and overall mortality in patients with Type II diabetes. Diabetologia 45:115-120

6. Schmid K (1989) Human plasma alpha 1-acid glycoproteinbiochemical properties, the amino acid sequence and the structure of the carbohydrate moiety, variants and polymorphism. Prog Clin Biol Res 300:7-22

7. Fournier T, Medjoubi N, Porquet D (2000) Alpha-1-acid glycoprotein. Biochim Biophys Acta 1482:157-171

8. Logdberg L, Wester L (2000) Immunocalins: a lipocalin subfamily that modulates immune and inflammatory responses. Biochim Biophys Acta 1482:284-297

9. Ross R (1999) Atherosclerosis - an inflammatory disease. N Engl J Med 340:115-126

10. Pickup JC, Crook MA (1998) Is type II diabetes mellitus a disease of the innate immune system? Diabetologia 41:12411248

11. McMillan DE (1989) Increased levels of acute-phase serum proteins in diabetes. Metabolism 38:1042-1046

12. Pickup JC, Mattock MB, Chusney GD, Burt D (1997) NIDDM as a disease of the innate immune system: association of acutephase reactants and interleukin-6 with metabolic syndrome X. Diabetologia 40:1286-1292

13. Duncan BB, Schmidt MI, Pankow JS et al (2003) Low-grade systemic inflammation and the development of type 2 diabetes: the atherosclerosis risk in communities study. Diabetes 52: 1799-1805

14. Ito S, Tsuda A, Momotsu T et al (1989) Urinary orosomucoid excretion rate in patients with non-insulin-dependent diabetes mellitus. Acta Endocrinol (Copenh) 120:584-590

15. Mogensen CE, Keane WF, Bennett PH et al (1995) Prevention of diabetic renal disease with special reference to microalbuminuria. Lancet 346:1080-1084
16. Engstrom G, Stavenow L, Hedblad B et al (2003) Inflammationsensitive plasma proteins, diabetes, and mortality and incidence of myocardial infarction and stroke: a population-based study. Diabetes 52:442-447

17. Schalkwijk CG, Poland DC, van Dijk W et al (1999) Plasma concentration of C-reactive protein is increased in type I diabetic patients without clinical macroangiopathy and correlates with markers of endothelial dysfunction: evidence for chronic inflammation. Diabetologia 42:351-357

18. Kuller LH, Tracy RP, Shaten J, Meilahn EN (1996) Relation of C-reactive protein and coronary heart disease in the MRFIT nested case-control study. Multiple Risk Factor Intervention Trial. Am J Epidemiol 144:537-547

19. Stehouwer CD, Gall MA, Twisk JW, Knudsen E, Emeis JJ, Parving HH (2002) Increased urinary albumin excretion, endothelial dysfunction, and chronic low-grade inflammation in type 2 diabetes: progressive, interrelated, and independently associated with risk of death. Diabetes 51:1157-1165

20. Vasson MP, Baguet JC, Arveiller MR, Bargnoux PJ, Giroud JP, Raichvarg D (1993) Serum and urinary alpha-1 acid glycoprotein in chronic renal failure. Nephron 65:299-303

21. Mogensen CE (1984) Microalbuminuria predicts clinical proteinuria and early mortality in maturity-onset diabetes. N Engl J Med 310:356-360

22. Neil A, Hawkins M, Potok M, Thorogood M, Cohen D, Mann J (1993) A prospective population-based study of microalbuminuria as a predictor of mortality in NIDDM. Diabetes Care 16:996-1003

23. Rossing P, Hougaard P, Borch-Johnsen K, Parving HH (1996) Predictors of mortality in insulin dependent diabetes: 10-year observational follow up study. Br Med J 313:779-784

24. Schmitz A, Vaeth M (1988) Microalbuminuria: a major risk factor in non-insulin-dependent diabetes. A 10-year follow-up study of 503 patients. Diabet Med 5:126-134

25. Messent JW, Elliott TG, Hill RD, Jarrett RJ, Keen H, Viberti GC (1992) Prognostic significance of microalbuminuria in insulindependent diabetes mellitus: a twenty-three-year follow-up study. Kidney Int 41:836-839

26. Borch-Johnsen K, Feldt-Rasmussen B, Strandgaard S, Schroll M, Jensen JS (1999) Urinary albumin excretion. An independent predictor of ischemic heart disease. Arterioscler Thromb Vasc Biol 19:1992-1997

27. Gerstein HC, Mann JF, Yi Q et al (2001) Albuminuria and risk of cardiovascular events, death, and heart failure in diabetic and nondiabetic individuals. JAMA 286:421-426

28. Hillege HL, Fidler V, Diercks GF et al (2002) Urinary albumin excretion predicts cardiovascular and noncardiovascular mortality in general population. Circulation 106:1777-1782

29. Harvey JN, Rizvi K, Craney L, Messenger J, Shah R, Meadows PA (2001) Population-based survey and analysis of trends in the prevalence of diabetic nephropathy in Type 1 diabetes. Diabet Med 18:998-1002

30. Mattock MB, Cronin N, Cavallo-Perin P et al (2001) Plasma lipids and urinary albumin excretion rate in Type 1 diabetes mellitus: the EURODIAB IDDM Complications Study. Diabet Med 18:59-67

31. Gall MA, Borch-Johnsen K, Hougaard P, Nielsen FS, Parving HH (1995) Albuminuria and poor glycemic control predict mortality in NIDDM. Diabetes 44:1303-1309

32. Gerstein HC, Mann JF, Pogue J et al (2000) Prevalence and determinants of microalbuminuria in high-risk diabetic and nondiabetic patients in the Heart Outcomes Prevention Evaluation Study. The HOPE Study Investigators. Diabetes Care 23: B35-B39

33. Christiansen MS, Blirup-Jensen S, Foged L, Larsen M, Magid E (2004) A particle-enhanced turbidimetric immunoassay for quantitative determination of orosomucoid in urine: development, validation and reference values. Clin Chem Lab Med 42:1168-1177 
34. Vittinghus E (1990) Preanalytical handling of stored urine samples, and measurement of beta 2-microglobulin, orosomucoid, albumin, transferrin and immunoglobulin $\mathrm{G}$ in urine by enzyme-linked immunosorbent assays (ELISA). Scand J Clin Lab Invest 50:843-849
35. Allen KV, Walker JD (2003) Microalbuminuria and mortality in long-duration type 1 diabetes. Diabetes Care 26:2389-2391

36. Fuller JH, Stevens LK, Wang SL (2001) Risk factors for cardiovascular mortality and morbidity: the WHO multinational study of vascular disease in diabetes. Diabetologia 44:S54-S64 